\title{
A connection between extracellular matrix and hormonal signals during the development of the human fetal adrenal gland
}

E. Chamoux ${ }^{1}$ M. Otis ${ }^{2}$ and N. Gallo-Payet ${ }^{2}$
${ }^{1}$ Rhumatologie/Immunologie, Centre de Recherches du CHUL

Québec, Canada

${ }^{2}$ Service Endocrinologie, Faculté de Médecine, Sherbrooke, Canada

Correspondence
1Present address
E. Chamoux
CRRI, Centre de Recherches du CHUL
Bloc T1-49
2705, Boulevard Laurier
Québec,
Canada G1V4G2
Fax: +1-418-654-2765
E-mail:
estelle.chamoux@crchul.ulaval.ca
2N. Gallo-Payet
Service Endocrinologie, Faculté de
Médecine 3001,
12éme avenue N,
Sherbrooke, Canada J1H 5N4

Presented at SIMEC 2004 (International Symposium on Extracellular Matrix), Angra dos Reis, RJ, Brazil, September 27-30, 2004.

Received February 16, 2005 Accepted July 20,2005

\section{Abstract}

The human adrenal cortex, involved in adaptive responses to stress, body homeostasis and secondary sexual characters, emerges from a tightly regulated development of a zone-specific secretion pattern during fetal life. Its development during fetal life is critical for the well being of pregnancy, the initiation of delivery, and even for an adequate adaptation to extra-uterine life. As early as from the sixth week of pregnancy, the fetal adrenal gland is characterized by a highly proliferative zone at the periphery, a concentric migration accompanied by cell differentiation (cortisol secretion) and apoptosis in the central androgen-secreting fetal zone. After birth, a strong reorganization occurs in the adrenal gland so that it better fulfills the newborn's needs, with aldosterone production in the external zona glomerulosa, cortisol secretion in the zona fasciculata and androgens in the central zona reticularis. In addition to the major hormonal stimuli provided by angiotensin II and adrenocorticotropin, we have tested for some years the hypotheses that such plasticity may be under the control of the extracellular matrix. A growing number of data have been harvested during the last years, in particular about extracellular matrix expression and its putative role in the development of the human adrenal cortex. Laminin, collagen and fibronectin have been shown to play important roles not only in the plasticity of the adrenal cortex, but also in cell responsiveness to hormones, thus clarifying some of the unexplained observations that used to feed controversies.
Key words

- Adrenal gland

- Development

- Human fetus

- Extracellular matrix

- Angiotensin II

- Adrenocorticotropin

\section{Introduction}

The human adrenal gland takes part in a number of major physiological processes including adaptation to stress, secondary sexual characters and fluid homeostasis, all of which require extremely tight regulation as well as a completely achieved fetal devel- opment (1). Strongly involved in feto-maternal exchanges, the fetal adrenal participates actively in the maintenance of pregnancy, as well as in the initiation of delivery (2). Adrenal steroids remain of critical importance during the newborn's development and adaptation to stress $(3,4)$. Moreover, an impaired development of the adrenal glands 
often results in sexual development disorders (male pseudohermaphrodism, female masculinization...), in an impaired fluid homeostasis ("loss of salt syndrome", sometimes leading to death) or even in mood disorders, depression, and metabolic disorders... (5-7).

In the fetal gland, the morphology, enzymatic apparatus and secretory patterns are completely different from those observed in adult subjects (2). The fetal gland is functionally active very early in the course of pregnancy and secretes a huge quantity of $\mathrm{C}^{19}$-steroids used by the placenta as estrogen precursors (8). In parallel to the ontogeny of secretory patterns, dictated by enzymatic machinery, the fetal adrenal exhibits a strong remodeling activity including proliferation, migration and apoptosis (9).

Angiotensin II (Ang II) and the adrenocorticotropic hormone (ACTH) are two major stimuli involved in the control of homeostasis and steroidogenic functions of the adrenals. Current data indicate that both hormones are also strongly involved in the fetal adrenal development $(10,11)$. However, signals provided by hormones alone cannot explain, by themselves, how the expression of zone-specific enzymes or hormone receptors is dictated. Indeed, a number of papers have reported the necessity of adrenal integrity to ensure adequate responses and secretions (12); others also reported large differences between what is observed in vitro and in vivo (13-15), suggesting that signals provided by the extracellular microenvironment are of critical importance for adrenal development and functions. For these reasons, a growing number of investigators have been interested in studying the role of extracellular matrix (ECM) in the morphology and steroidogenic functions of the adrenal gland. The present article is a review of studies that have contributed to a better understanding of interactions between hormonal and environmental signals in the adrenal gland, with a particular focus on fetal development.

\section{The human fetal adrenal development}

The development of the adrenal gland is very specific in human and great primates compared to non-primate species $(1,2)$. As early as the 6th week of pregnancy, some cells separate themselves from the pre-renal mesodermic outline and begin expressing the steroidogenic enzyme P450C17. As described in Figure 1, this enzymatic complex comprises a $17 \alpha$-hydroxylase and a $17-$ 20 lyase, thus being able to produce the androgenic steroid dehydroepiandrosterone (DHEA). Until the end of pregnancy, the fetal adrenal secretes DHEA and its sulfoconjugate DHEA/S in massive quantities (almost $200 \mathrm{mg}$ per day). This important adrenal $\mathrm{C}^{19}$-steroid secretion from the fetus plays a key role in feto-maternal communications, from the beginning of gestation until birth. Indeed, these steroids are aromatized by the placenta to form estrogens, essential for the maintenance of pregnancy $(8,16)$. As the fetal anlage proliferates (this part being called the fetal zone), some cell layers become non-proliferative and steroidogenically inactive at the periphery of the gland, where they form the definitive zone (17). At the same time, ectodermicderived cells detach from the neural crest and migrate to the future adrenal. At the 8th week, these pheochromoblasts are encapsulated with the adrenal and then penetrate successively the definitive and fetal zones to progressively colonize the deep center of the gland, where they will constitute the future medulla (18).

Until the 22nd week, the fetal zone remains extremely active in terms of androgen secretion. By the end of the second trimester of gestation (22-24 weeks), a morphologically distinct zone begins to be constituted at the interface of the definitive and fetal zones. This transitional zone acquires the enzyme 3ß-hydroxysteroid dehydrogenase (3ßHSD), thus allowing the fetus to secrete his 
own cortisol (2). Nothing is clear about the onset of 3ß-HSD or about the factors regulating it. Surely, this event is of importance to the fetus, in that the fetal cortisol is needed for a good maturation of numerous organs (lungs, gut, etc.) (19). On the other hand, an advanced expression of this enzyme, and consequently of cortisol secretion, may compromise pregnancy by inducing precocious abortion (20,21). Close to birth, the definitive zone acquires 3ß-HSD but this zone, being exempt of cytochrome P450C17, will secrete mineralocorticoids, which will be needed for extra-uterine life (22). During the entire course of pregnancy, an intense proliferative activity is observed at the periphery of the gland, followed by migration of the newly formed cells throughout the fetal cortex. In the course of their migration, cells differentiate, enlarge (becoming rich in steroid-filled lipid droplets) and finally die from apoptosis in the center of the gland $(9,23)$. Crucial for its active role during the whole pregnancy, the adrenal gland is one of the most voluminous of the fetal body. Shortly after birth, dramatic changes occur in the morphology of the newborn adrenal gland. Within only four months, the gland volume will be reduced 4-fold due to intense apoptotic activity in the fetal zone, which will rapidly disappear almost completely $(24,25)$. In the first years after birth, the definitive adrenal cortex will develop essentially from the definitive and transitional zones (those resembling the glomerulosa and fasciculata zone, respectively), whereas the reticularis zone is believed to be a remnant of the fetal zone. Figure 1 is a schematic representation of the morphological and functional development of the fetal adrenal.

\section{Hormonal control of adrenal development}

Few studies have described the very early factors involved in the human adrenal development. However, recent studies suggest that the major factors regulating adrenal functions in the first weeks are the transcription factors steroidogenic factor 1 and the nuclear hormone receptor DAX1 (26,27). Growth factors such as IGF-1 and IGF-2 are also important for the growth of the gland (28). In the adult as in the fetal gland, tight interrelations have been described between the me-

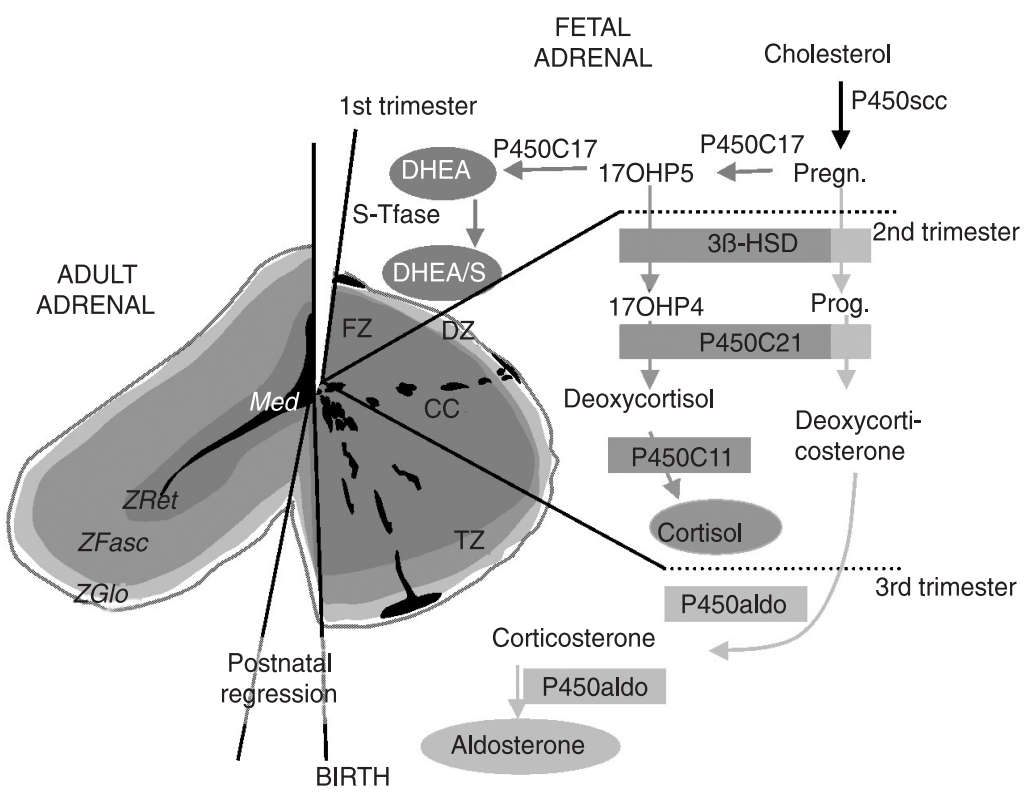

Figure 1. Ontogenesis of steroidogenic enzymes in the human fetal adrenal gland. This schematic representation is divided into portions showing the fetal adrenal gland (right) at the first, second and third trimesters of pregnancy, and the adult adrenal gland (left). During the first trimester, the fetal gland is composed of a definitive zone (DZ, light grey) and a fetal zone (FZ, dark grey). The fetal zone, expressing the P450C17 cytochrome, is responsible for massive secretion of DHEA and DHEA/S, used by the placenta as estrogen precursors. During the 2nd trimester, chromaffin cells (CC, black) originating from the neural crests migrate through the fetal cortex to progressively colonize the center of the gland to form the future medulla $(M e d)$. At the end of the 2 nd trimester, the newly constituted transitional zone (TZ, medium grey) acquires the enzyme 3B-HSD while the expression of P450C17 remains, thus allowing the production of fetal cortisol. Near birth, cells of the definitive zone which express only $3 B-H S D$, acquire the P450aldo and begin to secrete mineralocorticoids such as aldosterone. Shortly after birth, the fetal adrenal regresses strongly (mainly due to the regression of the fetal zone) and recovers progressively during the first years of extrauterine life. Finally, the adult adrenal gland is composed of the zona glomerulosa (ZGlo, light grey), zona fasciculata (ZFasc, medium grey) and zona reticularis (ZRet, dark grey) responsible for the production of mineralocorticoids (aldosterone), glucocorticoids (cortisol) and androgens (DHEA-DHEA/S), respectively. P450scc = cytochrome P450 side chain cleavage; Pregn. = pregnenolone; $\mathrm{P} 450 \mathrm{C} 17$ = cytochrome $\mathrm{P} 45017 \alpha$-hydroxylase, 17-20 lyase; 17OHP5 = 17-hydroxy-pregnenolone; DHEA/S = dehydroepiandrosteronesulfate; S-Tfase = DHEA sulfotransferase; $3 B-H S D=3 B$-hydroxysteroid dehydrogenase; Prog. = progesterone; $17 \mathrm{OHP} 4=17$-hydroxyprogesterone; $\mathrm{P} 450 \mathrm{C} 21$ = cytochrome $\mathrm{P} 450$ 21-hydroxylase; $\mathrm{P} 450 \mathrm{C} 11$ = cytochrome P450 11ß-hydroxylase; $\mathrm{P} 450$ aldo = cytochrome P450 aldosterone synthase. Adapted from Ref. 2. 
dulla and the cortex (29-31). During fetal development, the adrenal medulla is mostly controlled by neuropeptides like the corticotrophin-releasing factor, arginin-vasopressin, vaso-active intestinal polypeptide, or the pituitary adenylate cyclase activating peptide (32,33), and these neuropeptides may directly regulate steroidogenic functions of the fetal cortex (34). However, the cortical development of the adrenal is mostly controlled by the major hormones Ang II and ACTH (35). ACTH is certainly the most studied hormone with respect to cortical functions, but data are divergent concerning the early onset of adrenal responsiveness to ACTH secreted by the fetal hypophysis around the 8th week of pregnancy (36). At the 16th week, fetal zone cells appear to be under the control of this hormone while the definitive zone is not. However, even if ACTH is an important regulating factor of fetal adrenal functions during the entire pregnancy, it cannot explain the development of the fetal zone by itself. Indeed, anencephalic fetuses exhibit a fully constituted adrenal gland, even if reduced in the volume of the fetal zone (37). In vitro studies conducted on primary cultures of fetal adrenal cells show that ACTH immediately and strongly enhances the expression of both P450C17 and 33 -HSD $(38,39)$. However, these observations are not in full agreement with what is observed in vivo; indeed, if ACTH is present as early as the 8th week of gestation, the onset of 3ß-HSD expression does not occur before the 22nd week (2). This means that other factors are present in vivo which specifically inhibit the stimulating action of ACTH on fetal cortisol production and that these factors are absent in cell cultures. Results from our laboratory show a massive expression of the Ang II AT2 receptor in the whole fetal zone early in pregnancy (the earliest tested being at 14 weeks), whereas the expression of the AT1 receptor does not appear before the 16th week and is restricted to the definitive zone (40). We also reported that the strongly expressed AT2 receptor induces apoptosis in cells from the fetal zone, thus confirming the important apoptotic activity observed in the central part of the gland, a process involving cytoskeleton reorganization (41). Moreover, the AT2 receptor for Ang II, a fetal receptor disappearing from almost every tissue after birth, remains present in human and rat adrenal glands, correlating with the important remodeling observed in newborn adrenal glands (42). If hormonal signals seem to be of critical importance for morphological development and steroidogenic functions of the fetal adrenal, observations from many authors led us to believe that other signals may play an important role in these processes. In particular, considering the significant role of strict zone-specific patterns, signals provided by the extracellular microenvironment have been considered to be quite important for adrenal physiology for some years. The next part of this review will thus concentrate on the role of ECM in adrenal development.

\section{Control from the extracellular matrix}

First of all, the observations of Carsia et al. (12) in the rat adrenal gland showed that steroidogenic activities of the gland were directly influenced by its architecture. Older studies had also demonstrated that ECM was required to induce the expression of $11 ß$ HSD and 21-hydroxylase responsible for the end-processing of cortisol production in long-term cultures of bovine adrenal cells (43). Interestingly, the gene coding for tenascin-X overlaps the gene coding for the enzyme 21-hydroxylase in a duplicated, complex organization leading to the transcription of an adrenal-specific form of tenascin$X$ (44). Finally, the presence of thrombospondin-2 and laminin has been described in the bovine adult gland, where laminin is shown to play a potent chemoattractant role (45-47). 


\section{Extracellular matrix and the definitive zone}

In situ studies showed that the definitive zone, located at the periphery of the gland and sheltering cell proliferation, expresses laminin and collagen type IV as well as strong levels of integrins $\alpha_{1}, \alpha_{2}$, and $\beta_{1}$ chains (Figure 2 shows a schematic summary of ECM expression patterns observed in the human fetal adrenal gland) (48). Moreover, primary cultures of fetal adrenal cells conducted on laminin- or collagen-coated dishes showed an increased proliferation, results of an increased activity of definitive zone cells (49). Laminin also protected cells from apoptosis induced by the Ang II AT2 receptor, which is consistent with the weak level of apoptotic nuclei observed in the definitive zone (41).

In contrast, low levels of fibronectin were observed in the definitive zone, and $\alpha_{3}, \alpha_{4}$, $\alpha_{5}$, and $\alpha_{6}$ chains were not detected either. Logically, no proliferation was observed in cells cultured on fibronectin. Combined in situ and in vitro observations allow us to suggest that collagen and laminin, expressed in the definitive zone, contribute to maintain the high level of proliferation needed to ensure fetal adrenal growth.

\section{Extracellular matrix and differentiation of the transitional zone}

Our observations indicate that laminin expression is restricted to the definitive zone, no expression being detected either in the transitional or in the fetal zone. In contrast, collagen type IV is abundant in the transitional zone where an increased level of integrin $\alpha_{2}$ chain is also detected, as well as $\alpha_{1}$ and $\beta_{1}$ chains. In vitro studies conducted on collagen IV showed that cortisol secretion in response to hormones was strongly enhanced. Indeed, cortisol secreted in response to ACTH was doubled when cells were cultured on collagen rather than on mere plastic, an ef- fect reflected by an increased expression of the limiting enzyme 3ß-HSD. A similar effect was observed for cortisol secreted in response to Ang II, via its AT1 receptor that is already known to induce such secretions in the adult gland (50).

Fibronectin exhibits a gradient of expression more concentrated when going from the periphery to the center of the gland, and was thus present in the transitional zone. Completely absent from the definitive zone, the integrin $\alpha_{3}$ chain was observed in the transitional and fetal zone. Even if this integrin $\alpha$ chain is not a high-affinity receptor for fibronectin, it has been described to bind it and to induce a signal when no other receptor is available for fibronectin. Interestingly, fetal adrenal cells cultured on fibronectin failed to respond to ACTH in terms of cortisol secretion, this matrix reducing significantly cortisol levels compared to those observed on mere plastic. Fibronectin also abolished the expression of 3ß-HSD nor-

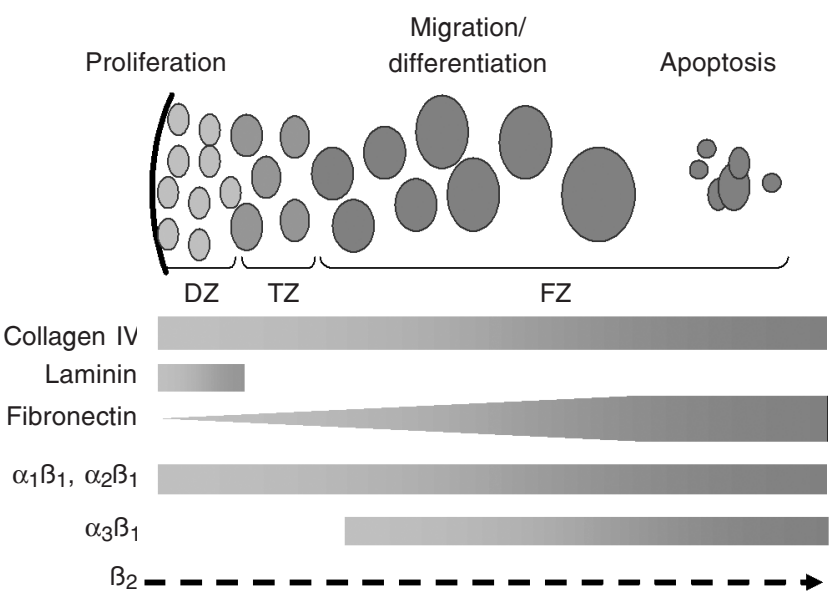

Figure 2. Schematic representation of extracellular matrix components expressed in the human fetal adrenal gland. Cells of the fetal adrenal cortex proliferate in the definitive zone (DZ, light grey), then enter the transitional zone (TZ, medium grey) where they begin their migration throughout the whole fetal cortex, differentiate progressively into fetal zone cells (FZ, dark grey) and finally die from apoptosis in the center of the fetal zone. Collagen IV is expressed throughout the fetal cortex, as also are integrins $\alpha_{1}, \alpha_{2}$ and $B_{1}$ chains. Laminin expression is restricted to the definitive zone, whereas fibronectin exhibits a gradient of expression more concentrated in the center of the gland. The integrin $\alpha_{3}$ is expressed only in the fetal zone, and a strong expression of the $B_{2}$ chain has been described on the surface of chromaffin cells. 
mally seen on plastic after ACTH stimulation. It should be noted that, at this stage of development (18 weeks), the human fetal adrenal gland is not supposed to secrete cortisol by itself. One of our hypotheses is that the expression of fibronectin in the transitional zone contributes to attenuate cell responsiveness to $\mathrm{ACTH}$, thus explaining the controversy about the onset of 3B-HSD expression and cortisol secretion by the fetus, a critical point for avoiding precocious abortion.

\section{Extracellular matrix and the development of the fetal zone}

The main characteristic of the fetal zone is an abundant expression of collagen, and even more of fibronectin. The integrins $\alpha_{1}$, $\alpha_{2}, \alpha_{3}$ and $\beta_{1}$ chains are also present, thus allowing cells to bind collagen and laminin to a lesser extent (51). This zone is known to secrete massive amounts of DHEA and DHEA/S during the entire course of pregnancy, thus playing an essential role in its maintenance by providing estrogen precursors used by the placenta (8). Primary cultures of fetal adrenal cells conducted on fibronectin exhibited a high level of apoptosis, either basal or stimulated by the Ang II AT2 receptor. Compared to plastic or collagen, fibronectin also strongly enhanced DHEA and DHEA/S secretion, either at a basal level or even more when stimulated by ACTH. Reflecting this secretion pattern, the expression of $\mathrm{P} 450 \mathrm{C} 17$ was increased in adrenal cells cultured on fibronectin rather than on other matrices. The respective roles of fibronectin and AT2 receptors seem to be strongly imbricated and to act complementarily. However, studies conducted on other models show that stimulation of the AT2 receptor induces fibronectin expression (52). On the basis of these observations, one can suggest that fibronectin plays an important role in setting up the characteristics of the fetal zone, e.g., strong expression of
P450C17, massive secretions of DHEA/ DHEA/S and a high level of apoptosis.

\section{Connections between extracellular matrix and hormonal signals}

Ang II and ECM have been shown to be strikingly interconnected in a number of models including heart or vasculature (53). Both of them play an important role in the architecture of the cytoskeleton and they share a number of common pathways. In the particular case of the adrenal gland, Ang II induces a strong reorganization of the cytoskeleton, which is required to generate a signaling cascade leading to the production of aldosterone (54). Ang II has also been shown to activate cytoskeleton-associated proteins such as RhoA, Pyk2, the focal adhesion kinase, paxilin and/or tensin (55). A specific stimulation of the Ang II AT2 receptor also induces strong cytoskeletal reorganization $(41,56)$ and the production of fibronectin or thrombospondin 1 (52), whereas stimulation of the AT1 receptor leads to collagen production (53). The ECM has been shown in numerous models to generate cytoskeletal reorganization after integrin binding and aggregation in focal adhesions, resulting in survival, differentiation and/or migration pathways. The observations of the human fetal adrenal gland suggest that cell migration from the periphery to the center of the gland may be driven by collagen IV and/ or fibronectin. In particular, cells from the fetal zone expressing the $\alpha_{1} \beta_{1}$ integrins may use collagen IV as their substrate for migration, as described in other models (57). In contrast, chromaffin cells expressing the $\beta_{2}$ integrin subunit may, like inflammatory cells, bind fibronectin to ensure their migration (58). Based on the observations described above, one may also suggest that the ECM could be involved in the expression of the Ang II AT2 receptor. Indeed, even though this receptor was previously shown to be strongly expressed in vivo, its expression 
falls dramatically a few hours after cell culture (40), indicating that the extracellular microenvironment is critical for its turnover. Both Ang II AT2 receptor and fibronectin promote apoptosis of fetal zone cells. Moreover, in the rat adrenal gland, the binding of fibronectin to its specific integrins generates signals shared by Ang II, like an elevation of intracellular calcium (59).

The relationships between ACTH and ECM have been less well studied than those of Ang II. Like Ang II, the ACTH signaling cascade has been shown to involve the actin cytoskeleton $(54,60)$. The differences observed by several groups between the activity of steroidogenic enzymes in vitro versus in vivo strongly suggest that the environment influences cell responsiveness towards this hormone. In particular, collagen IV seems to potentiate cell responsiveness to ACTH in terms of cortisol secretion and 3ßHSD expression.

All of these data suggest that the ECM and hormones are strikingly interconnected and share common pathways involved in the control of adrenal gland activities. We have tried to review what is known about relationships existing between ECM and the major hormones involved in adrenal development, e.g., Ang II and ACTH. All the results reviewed and commented here show that the hormonal control of the adrenal gland is strongly influenced by the ECM, and consequently allow us to better understand fetal adrenal physiology and development.

\section{References}

1. McNicol A (1992). The human adrenal gland: aspects of structure, function and pathology. In: James VHT (Editor), The Adrenal Gland. Raven Press Ltd., New York.

2. Mesiano S \& Jaffe RB (1997). Developmental and functional biology of the primate fetal adrenal cortex. Endocrine Reviews, 18: 378-401.

3. Salzmann C, Otis M, Long $\mathrm{H}$ et al. (2004). Inhibition of steroidogenic response to adrenocorticotropin by leptin: implications for the adrenal response to maternal separation in neonatal rats. Endocrinology, 145: 1810-1822.

4. Walker CD, Salzmann C, Long $\mathrm{H}$ et al. (2004). Direct inhibitory effects of leptin on the neonatal adrenal and potential consequences for brain glucocorticoid feedback. Endocrine Research, 30: 837844.

5. White PC (1994). Genetic diseases of steroid metabolism. Vitamins and Hormones, 49: 131-195.

6. White PC (2001). Congenital adrenal hyperplasias. Best Practice and Research. Clinical Endocrinology and Metabolism, 15: 17-41.

7. White PC (2004). Aldosterone synthase deficiency and related disorders. Molecular and Cellular Endocrinology, 217: 81-87.

8. Pepe GJ \& Albrecht ED (1995). Actions of placental and fetal adrenal steroid hormones in primate pregnancy. Endocrine Reviews, 16: 608-648.

9. Nussdorfer G (1986). The fetal adrenal cortex. International Review of Cytology, 98: 211-249.

10. Di Blasio A, Fujii D, Yamamoto M et al. (1990). Maintenance of cell proliferation and steroidogenesis in cultured human fetal adrenal cells chronically exposed to adrenocorticotropic hormone: rationalization of in vitro and in vivo findings. Biology of Reproduction, 42: 683-691.

11. Voutilainen R, Ilveskami V \& Miettinen P (1991). Low expression of 3b-hydroxy-5-ene steroid dehydrogenase gene in human fetal adrenals in vivo: adrenocorticotropin and protein kinase C-dependent regulation in adrenocortical cultures. Journal of Clinical Endocrinology and Metabolism, 72: 761-767.

12. Carsia RV, Tilly KI \& Tilly JL (1997). Hormonal modulation of apoptosis in the rat adrenal gland in vitro is dependent on structural integrity. Endocrine, 7: 377-381.

13. Fujieda K, Faiman C, Feyes Fl et al. (1982). The control of steroidogenesis by human fetal adrenal cells in tissue culture. IV. The effect of exposure to placental steroids. Journal of Clinical Endocrinology and Metabolism, 54: 89-94.

14. Fujieda K, Faiman C, Reyes FI et al. (1981). The control of steroidogenesis by human fetal adrenal cells in tissue culture. III. The effects of various hormonal peptides. Journal of Clinical Endocrinology and Metabolism, 53: 690-693.

15. McDonald T \& Nathanielsz P (1998). The involvement of innervation in the regulation of fetal adrenal steroidogenesis. Hormone and Metabolic Research, 30: 297-302.

16. Pepe GJ \& Albrecht ED (1990). Regulation of the primate fetal adrenal cortex. Endocrine Reviews, 11: 151-176.

17. Wolkersdorfer $G$ \& Bornstein $S$ (1998). Tissue remodeling in the adrenal gland. Biochemical Pharmacology, 56: 163-171.

18. Bocian-Sobkowska J, Wozniak W, Malendowicz L et al. (1996). Stereology of human fetal adrenal medulla. Histology and Histopathology, 11: 389-393.

19. Liggins $G$ (1976). Adrenocortical-related maturational events in the fetus. American Journal of Obstetrics and Gynecology, 126: 931941.

20. Ochedalski T \& Lachowicz A (2004). Maternal and fetal hypothalamo-pituitary-adrenal axis: different response depends upon the mode of parturition. Neuroendocrinology Letters, 25: 278-282.

21. Ochedalski T, Zylinska K, Laudanski T et al. (2001). Corticotrophin- 
releasing hormone and $\mathrm{ACTH}$ levels in maternal and fetal blood during spontaneous and oxytocin-induced labour. European Journal of Endocrinology, 144: 117-121.

22. White PC (1997). Abnormalities of aldosterone synthesis and action in children. Current Opinion in Pediatrics, 9: 424-430.

23. Toth IE, Szabo D \& Bruckner GG (1997). Lipoproteins, lipid droplets, lysosomes, and adrenocortical steroid hormone synthesis: morphological studies. Microscopy Research and Technique, 36: 480-492.

24. Winter J (1992). Fetal and neonatal adrenocortical development. In: James $\mathrm{V}$ (Editor), The Adrenal Cortex. Raven Press, New York, 87104.

25. Winter J (1985). The Adrenal Cortex in the Fetus and Neonate. Butterworths, London, UK.

26. Hanley NA, Rainey WE, Wilson DI et al. (2001). Expression profiles of SF-1, DAX1, and CYP17 in the human fetal adrenal gland: potential interactions in gene regulation. Molecular Endocrinology, 15: 5768.

27. Ozisik G, Achermann JC, Meeks JJ et al. (2003). SF1 in the development of the adrenal gland and gonads. Hormone Research, 59 (Suppl 1): 94-98.

28. Mesiano S \& Jaffe R (1997). Role of growth factors in the developmental regulation of the human fetal adrenal cortex. Steroids, 62: 62-72.

29. Ehrhart-Bornstein M, Breidert M, Guadanucci P et al. (1997). 17 $\alpha$ Hydroxylase and chromogranin A in 6th week human fetal adrenals. Hormone and Metabolic Research, 29: 30-32.

30. Ehrhart-Bornstein M, Hinson J, Bornstein S et al. (1998). Intraadrenal interactions in the regulation of adrenocortical steroidogenesis. Endocrine Reviews, 19: 101-143.

31. Gallo-Payet N, Pothier P \& Isler H (1987). On the presence of chromaffin cells in the adrenal cortex: their possible role in adrenocortical function. Biochemistry and Cell Biology, 65: 588-592.

32. Payet MD, Bilodeau L, Breault $L$ et al. (2003). PAC1 receptor activation by PACAP-38 mediates $\mathrm{Ca}^{2+}$ release from a cAMP-dependent pool in human fetal adrenal gland chromaffin cells. Journal of Biological Chemistry, 278: 1663-1670.

33. Yon L, Breault L, Contesse V et al. (1998). Localization, characterization, and second messenger coupling of pituitary adenylate cyclase-activating polypeptide receptors in the fetal human adrenal gland during the second trimester of gestation. Journal of Clinical Endocrinology and Metabolism, 83: 1299-1305.

34. Breault L, Yon L, Montero M et al. (2000). Occurrence and effect of PACAP in the human fetal adrenal gland. Annals of the New York Academy of Sciences, 921: 429-433.

35. Leavitt MG, Albrecht ED \& Pepe GJ (1999). Development of the baboon fetal adrenal gland: regulation of the ontogenesis of the definitive and transitional zones by adrenocorticotropin. Journal of Clinical Endocrinology and Metabolism, 84: 3831-3835.

36. Winters A, Oliver C, Colston C et al. (1974). Plasma ACTH levels in the human fetus and neonate as related to age and parturition. Journal of Clinical Endocrinology and Metabolism, 39: 269-273.

37. Bocian-Sobkowska J, Malendowicz L \& Wozniak W (1997). Comparative stereological studies on zonation and cellular composition of adrenal glands of normal and anencephalic human fetuses. II. Cellular composition of the gland. Histology and Histopathology, 12: 391-399.

38. Coulter CL, Goldsmith PC, Mesiano S et al. (1996). Functional maturation of the primate fetal adrenal in vivo. II. Ontogeny of corticosteroid synthesis is dependent upon specific zonal expression of 3 beta-hydroxysteroid dehydrogenase/isomerase. Endocrinology, 137: 4953-4959.
39. Stocco D \& Clark B (1996). Regulation of the acute production of steroids in steroidogenic cells. Endocrine Reviews, 17: 221-244.

40. Breault L, LeHoux JG \& Gallo-Payet N (1996). The angiotensin AT2 receptor is present in the human fetal adrenal gland throughout the second trimester of gestation. Journal of Clinical Endocrinology and Metabolism, 81: 3914-3922.

41. Chamoux E, Breault L, LeHoux J-G et al. (1999). Involvement of the angiotensin II type 2 receptor in apoptosis during human fetal adrenal development. Journal of Clinical Endocrinology and Metabolism, 84: 4722-4730.

42. Shanmugam S, Llorens-Cortes C, Clauser E et al. (1995). Expression of angiotensin II AT2 receptor mRNA during development of rat kidney and adrenal gland. American Journal of Physiology, 268 : F922-F930.

43. Cheng $C$ \& Hornsby $P$ (1992). Expression of 11 beta-hydroxylase and 21-hydroxylase in long-term cultures of bovine adrenocortical cells requires extracellular matrix factors. Endocrinology, 130: 28832889.

44. Tee MK, Thomson AA, Bristow J et al. (1995). Sequences promoting the transcription of the human XA gene overlapping P450c21A correctly predict the presence of a novel, adrenal-specific, truncated form of tenascin-X. Genomics, 28: 171-178.

45. Danik M, Chinn AM, Lafeuillade B et al. (1999). Bovine thrombospondin-2: complete complementary deoxyribonucleic acid sequence and immunolocalization in the external zones of the adrenal cortex. Endocrinology, 140: 2771-2780.

46. Pellerin S, Keramidas M, Chambaz EM et al. (1997). Expression of laminin and its possible role in adrenal cortex homeostasis. Endocrinology, 138: 1321-1327.

47. Pellerin S, Lafeuillade B, Chambaz EM et al. (1994). Distinct effects of thrombospondin-1 and CISP/thrombospondin-2 on adrenocortical cell spreading. Molecular and Cellular Endocrinology, 106: 181-186.

48. Chamoux E, Bolduc L, Lehoux JG et al. (2001). Identification of extracellular matrix components and their integrin receptors in the human fetal adrenal gland. Journal of Clinical Endocrinology and Metabolism, 86: 2090-2098.

49. Chamoux E, Narcy A, Lehoux JG et al. (2002). Fibronectin, laminin, and collagen IV as modulators of cell behavior during adrenal gland development in the human fetus. Journal of Clinical Endocrinology and Metabolism, 87: 1819-1828.

50. Balla T, Baukal A, Eng $S$ et al. (1991). Angiotensin II receptor subtypes and biological responses in the adrenal cortex and medulla. Molecular Pharmacology, 40: 401-406.

51. Kuhn K \& Eble J (1994). The structural bases of integrin-ligand interactions. Trends in Cell Biology, 4: 256-261.

52. Fischer JW, Stoll M, Hahn AW et al. (2001). Differential regulation of thrombospondin-1 and fibronectin by angiotensin II receptor subtypes in cultured endothelial cells. Cardiovascular Research, 51: 784-791.

53. Brilla C, Scheer C \& Rupp H (1997). Renin-angiotensin system and myocardial collagen matrix: modulation of cardiac fibroblast function by angiotensin II type 1 receptor antagonism. Journal of Hypertension, 15 (Suppl): S13-S19.

54. Cote M, Payet MD, Dufour MN et al. (1997). Association of the G protein $\mathrm{a}_{\mathrm{q}} / \mathrm{a}_{11}$-subunit with cytoskeleton in adrenal glomerulosa cells: role in receptor-effector coupling. Endocrinology, 138: 3299-3307.

55. Haendeler J \& Berk BC (2000). Angiotensin II mediated signal transduction. Important role of tyrosine kinases. Regulatory Peptides, 95: 1-7.

56. Laflamme L, De Casparo M, Gallo JM et al. (1996). Angiotensin II induction of neurite outgrowth by AT2 receptors in NG108-15 cells. 
Journal of Biological Chemistry, 271: 22729-22735.

57. Gotwals P, Chi-Rosso G, Lindner V et al. (1996). The alpha1beta1 integrin is expressed during neointima formation in rat arteries and mediates collagen matrix reorganization. Journal of Clinical Investigation, 97: 2469-2477.

58. Chavakis E, Aicher A, Heeschen C et al. (2005). Role of \{beta\}2integrins for homing and neovascularization capacity of endothelial progenitor cells. Journal of Experimental Medicine, 201: 63-72.
59. Campbell S, Otis M, Cote M et al. (2003). Connection between integrins and cell activation in rat adrenal glomerulosa cells: a role for Arg-Gly-Asp peptide in the activation of the p42/p44(mapk) pathway and intracellular calcium. Endocrinology, 144: 1486-1495.

60. Cote M, Payet MD \& Gallo-Payet N (1997). Association of alpha Ssubunit of the GS protein with microfilaments and microtubules: implication during adrenocorticotropin stimulation in rat adrenal glomerulosa cells. Endocrinology, 138: 69-78. 